\title{
Existence theorems for nonlinear second-order three-point boundary value problems involving the distributional Henstock-Kurzweil integral
}

\author{
Xuexiao You ${ }^{\mathrm{a}, \mathrm{b}}$, Wei Liu ${ }^{\mathrm{c}}$, Guoju Ye ${ }^{\mathrm{c}}$, Dafang Zhao ${ }^{\mathrm{a}, \mathrm{c}, *}$ \\ a School of Mathematics and Statistics, Hubei Normal University, Huangshi, Hubei 435002, P. R. China. \\ ${ }^{b}$ College of Computer and Information, Hohai University, Nanjing, Jiangsu 210098, P. R. China. \\ ${ }^{c}$ College of Science, Hohai University, Nanjing, Jiangsu 210098, P. R. China.
}

Communicated by Y. Z. Chen

\begin{abstract}
In this paper, we are concerned with the existence of solutions for a second-order three-point nonlinear boundary value problems involving the distributional Henstock-Kurzweil integral. By using the Leray-Schauder nonlinear alternative, we achieve some results which are the generalizations of the previous results in the literatures. (C)2017 All rights reserved.
\end{abstract}

Keywords: Distributional Henstock-Kurzweil integral, nonlinear boundary value problems, distributional derivative, Leray-Schauder nonlinear alternative.

2010 MSC: 34B15, 26A39, 46G12.

\section{Introduction and preliminaries}

In recent years, many authors have studied three-point boundary value problems for second order differential equations in $[1,3,4,8,11-13,15-18,21,22,25]$. Such problems have potential applications in chemistry, physics, biology, etc. In this article, using the Leray-Schauder nonlinear alternative, we establish the existence of solution of the second order differential equation of the type

$$
-x^{\prime \prime}=f+g(t, x), \quad t \in[0,1],
$$

subject to the three-point boundary condition

$$
x(0)=\beta x^{\prime}(0), x^{\prime}(1)+x^{\prime}(\eta)=0,
$$

where $x^{\prime \prime}, x^{\prime}$ stand for the distributional derivative of the real function $x \in C^{1}[0,1], C^{1}[0,1]$ denotes the space where first order derivatives of the functions are continuous, $f$ is a distribution (generalized function), $\beta$ is a constant and $\eta \in[0,1]$.

\footnotetext{
*Corresponding author

Email addresses: youxuexiao@126.com (Xuexiao You), liuw626@hhu.edu.cn (Wei Liu), yegj@hhu.edu.cn (Guoju Ye), dafangzhao@163. com (Dafang Zhao)

doi:10.22436/jnsa.010.04.42
} 
This study is motivated by Bullen, Sarkhel's results in [5] in which the differential equations involving the approximate derivatives was considered. It is well-known that the notion of a distributional derivative is very general, including ordinary derivatives and approximate derivatives. The purpose of this paper is that use the distributional derivatives instead of the usual derivatives to discuss the second order nonlinear boundary value problems.

This paper is organized as follows. In Section 2, we introduce fundamental concepts and basic results of the distributional Henstock-Kurzweil integral. In Section 3, we apply the Leray-Schauder nonlinear alternative to verify the existence results of the problem (1.1)-(1.2). In Section 4, we give two examples to illustrate Theorem 3.3 and Corollary 3.4 in this paper.

\section{The distributional Henstock-Kurzweil integral}

In this section, the definition of the distributional Henstock-Kurzweil integral and its main properties needed in this paper are presented.

Define the space

$$
\mathrm{C}_{\mathrm{c}}^{\infty}=\left\{\phi: \mathbb{R} \rightarrow \mathbb{R} \mid \phi \in \mathrm{C}^{\infty} \text { and } \phi \text { has compact support in } \mathbb{R}\right\},
$$

where the support of a function $\phi$ is the closure of the set on which $\phi$ does not vanish, denoted by $\operatorname{supp}(\phi)$. A sequence $\left\{\phi_{\mathrm{n}}\right\} \subset \mathrm{C}_{\mathcal{c}}^{\infty}$ converges to $\phi \in \mathrm{C}_{\mathcal{c}}^{\infty}$ if there is a compact set $\mathrm{K}$ such that all $\phi_{\mathrm{n}}$ have supports in $K$ and for every $m \in \mathbb{N}$ the sequence of $m$-th derivatives $\phi_{\mathfrak{n}}^{(m)}$ converges to $\phi^{(m)}$ uniformly on $K$. Let $C_{c}^{\infty}$ be endowed with this convergence property and denote it by $\mathcal{D}$. Also, $\phi$ is called test function if $\phi \in \mathcal{D}$. Distributions are defined to be continuous linear functionals on $\mathcal{D}$. The space of distributions is denoted by $\mathcal{D}^{\prime}$, which is the dual space of $\mathcal{D}$. That is, if $f \in \mathcal{D}^{\prime}$ then $f: \mathcal{D} \rightarrow \mathbb{R}$ is a linear functional and we write $\langle f, \phi\rangle \in \mathbb{R}$, for $\phi \in \mathcal{D}$.

For all $f \in \mathcal{D}^{\prime}$, we recall that the distributional derivative $\mathrm{Df}$ of $\mathrm{f}$ is a distribution satisfying $\langle\mathrm{Df}, \phi\rangle=$ $-\left\langle f, \phi^{\prime}\right\rangle$, where $\phi$ is a test function and $\phi^{\prime}$ is the ordinary derivative of $\phi$. With this definition, all distributions have derivatives of all orders and each derivative is a distribution.

Let $(a, b)$ be an open interval in $\mathbb{R}$. We define

$$
\mathcal{D}(\mathrm{a}, \mathrm{b})=\left\{\phi: \mathbb{R} \rightarrow \mathbb{R} \mid \phi \in \mathrm{C}^{\infty} \text { and } \phi \text { has compact support in }(\mathrm{a}, \mathrm{b})\right\} .
$$

Of course, $\mathcal{D}(a, b)$ is endowed with the above convergence property. The dual space of $\mathcal{D}(a, b)$ is denoted by $\mathcal{D}^{\prime}(a, b)$. In this case, if $a=-\infty, b=+\infty$, then $\mathcal{D}(a, b)=\mathcal{D}$ and $\mathcal{D}^{\prime}(a, b)=\mathcal{D}^{\prime}$.

Define $C[a, b]$ to be the space of continuous functions on $[a, b]$, and

$$
\mathrm{C}_{0}=\{\mathrm{F} \in \mathrm{C}[\mathrm{a}, \mathrm{b}] \mid \mathrm{F}(\mathrm{a})=0\} .
$$

Note that $\mathrm{C}_{0}$ is a Banach space with the uniform norm $\|\mathrm{F}\|_{\infty}=\max _{[a, b]}|\mathrm{F}|$.

We are now able to present the definition of the $\mathrm{D}_{\mathrm{HK}}$-integral.

Definition 2.1 ([24, Definition 2.1]). A distribution $f$ is distributionally Henstock-Kurzweil integrable or briefly $\mathrm{D}_{\mathrm{HK}}$-integrable on $[\mathrm{a}, \mathrm{b}]$ if $\mathrm{f}$ is the distributional derivative of a continuous function $\mathrm{F} \in \mathrm{C}_{0}$.

The space of $\mathrm{D}_{\mathrm{HK}}$-integrable distributions is defined by

$$
D_{H K}=\left\{f \in \mathcal{D}^{\prime}(a, b) \mid f=D F \text { for some } F \in C_{0}\right\} .
$$

With this definition, if $f \in D_{H K}$ then, for all $\phi \in \mathcal{D}(a, b)$,

$$
\langle f, \phi\rangle=\langle D F, \phi\rangle=-\left\langle F, \phi^{\prime}\right\rangle=-\int_{a}^{b} F \phi^{\prime} .
$$

The second equality holds because $F$ and $\phi$ are continuous on $(a, b)$ and $\phi^{\prime}$ has compact support in $(a, b)$. The integral in the last equality exists as a Riemann integral for the same reason. 
For convenience, we write $\int_{a}^{b} f=F(b)$, where $F$ is called the primitive of $f$ and, unless otherwise stated,

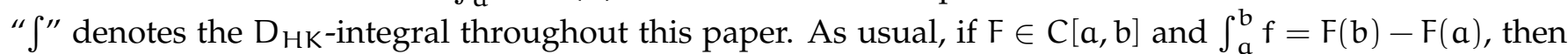
the function $F$ is a primitive of $f$. Notice that if $f \in D_{H K}$ then $f$ has many primitives in $C[a, b]$, all differing by a constant, but $f$ has exactly one primitive in $C_{0}$.

With this definition, this integral comprises Riemann, Lebesgue, Henstock-Kurzweil, Perron, Denjoy, and improper integrals as special cases ([10, 14, 19, 20, 23]).

Remark 2.2. Integrals defined in the same way have also been proposed in other papers. For example, Ang et al. [2] defined it in the plane and called it the G-integral, and Talvila [23] defined the $A_{C}$-integral on the extended real line. In that case of integration over one-dimensional interval, these two integrals coincide.

The following result is known as the Fundamental Theorem of Calculus.

Lemma 2.3 ([23, Theorem 4]).

(a) Let $f \in D_{H K}$ and $F(x)=\int_{a}^{x} f$. Then $F \in C_{0}$ and $D F=f$.

(b) Let $\mathrm{F} \in \mathrm{C}[\mathrm{a}, \mathrm{b}]$. Then $\int_{\mathrm{a}}^{\mathrm{x}} \mathrm{DF}=\mathrm{F}(\mathrm{x})-\mathrm{F}(\mathrm{a})$ for all $\mathrm{x} \in[\mathrm{a}, \mathrm{b}]$.

For $F, G \in C_{0}$, we say that $F \leqslant G$ if and only if $F(x) \leqslant G(x)$ for all $x \in[a, b]$. Similarly, for $f, g \in D_{H K}$, we say that $f \preceq g$ (or $g \succeq f$ ) if and only if

$$
\int_{\mathrm{I}} \mathrm{f} \leqslant \int_{\mathrm{I}} \mathrm{g} \quad \forall \mathrm{I}:=[\mathrm{c}, \mathrm{d}] \subset[\mathrm{a}, \mathrm{b}] .
$$

Denote $D_{H K+}=\left\{f \in D_{H K}: f \succeq 0\right\}$. The following lemma will be needed later.

Lemma 2.4 ([2, Corollary 1]). If $f_{1}, f_{2}, f_{3} \in \mathcal{D}^{\prime}(a, b), f_{1} \preceq f_{2} \preceq f_{3}$, and if $f_{1}$ and $f_{3}$ are $D_{H K}$-integrable, then $f_{2}$

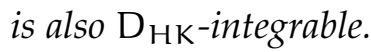

Let $f \in D_{H K}, F \in C_{0}$ with DF $=f$. Then, under the Alexiewicz norm

$$
\|f\|=\|F\|_{\infty}=\max _{[a, b]}|F|,
$$

$D_{H K}$ is a Banach space (see [23, Theorem 2]).

We say that a sequence $\left\{f_{n}\right\} \subset D_{H K}$ converges strongly to $f \in D_{H K}\left(\right.$ or $f_{n} \rightarrow f$ in $D_{H K}$ ) if $\left\|f_{n}-f\right\| \rightarrow 0$ as $n \rightarrow \infty$. In addition, we have a convergence theorem, which play an important role in our proof.

Lemma 2.5 ([2, Corollary 5, Dominated convergence theorem]). Let $\left\{\mathrm{f}_{\mathfrak{n}}\right\}_{\mathfrak{n}=0}^{\infty}$ be a sequence in $\mathrm{D}_{\mathrm{HK}}$ such that $\mathrm{f}_{\mathrm{n}} \rightarrow \mathrm{f}$ in $\mathcal{D}^{\prime}$. Suppose there exist $\mathrm{f}_{-}, \mathrm{f}_{+} \in \mathrm{D}_{\mathrm{HK}}$ satisfying $\mathrm{f}_{-} \preceq \mathrm{f}_{\mathrm{n}} \preceq \mathrm{f}_{+}$, for all $\mathrm{n} \in \mathbb{N}$. Then $\mathrm{f} \in \mathrm{D}_{\mathrm{HK}}$ and $\lim _{n \rightarrow \infty} \int_{a}^{b} f_{n}=\int_{a}^{b} f$.

If $g:[a, b] \rightarrow \mathbb{R}$, its variation is $V g=\sup \sum_{n}\left|g\left(t_{n}\right)-g\left(s_{n}\right)\right|$, where the supremum is taken over every sequence $\left\{\left(t_{n}, s_{n}\right)\right\}$ of disjoint intervals in $[a, b]$. If $V g<\infty$ then $g$ is called a function with bounded variation. Denote the set of functions with bounded variation by $\mathcal{B} \mathcal{V}$. As it is known that the dual space of $D_{H K}$ is $\mathcal{B V}$ (see details in [23]), we have the next result.

Lemma 2.6 ([23, Definition 6, Integration by parts]). Let $f \in D_{H K}$, and $g \in \mathcal{B V}$. Define $f g=D H$, where $\mathrm{H}(\mathrm{t})=\mathrm{F}(\mathrm{t}) \mathrm{g}(\mathrm{t})-\int_{\mathrm{a}}^{\mathrm{t}} \mathrm{Fdg}$. Then $\mathrm{fg} \in \mathrm{D}_{\mathrm{HK}}$ and

$$
\int_{a}^{b} f g=F(b) g(b)-\int_{a}^{b} F d g .
$$

Lemma 2.7 ([23, Theorem 7, Hölder inequality]). Let $\mathrm{f} \in \mathrm{D}_{\mathrm{HK}}$. If $\mathrm{g} \in \mathcal{B} \mathcal{V}$, then

$$
\left|\int_{a}^{b} f g\right| \leqslant 2\|F\|\|g\|_{\mathcal{B} \mathcal{~} .}
$$


The next convergence theorem is a direct consequence of the Hölder inequality.

Lemma 2.8. Let $\left\{f_{n}\right\} \subset D_{H K}$. If there exists $f \in D_{H K}$ such that $f_{n} \rightarrow f$ in $D_{H K}$, i.e., $\left\|f_{n}-f\right\| \rightarrow 0$ as $n \rightarrow+\infty$, then for all $\mathrm{g} \in \mathcal{B} V, \mathrm{f}_{\mathrm{n}} \mathrm{g} \rightarrow \mathrm{fg}$ and

$$
\lim _{n \rightarrow+\infty} \int_{a}^{b} f_{n} g=\int_{a}^{b} f g
$$

\section{Main results}

In this section, we firstly assume that $f, g$ satisfy the following assumptions:

$\left(\mathrm{H}_{1}\right) \mathrm{g}(\mathrm{x}, \mathrm{t})$ is $\mathrm{HK}$-integrable with respect to $\mathrm{t}$ for all $\mathrm{x} \in \mathrm{C}^{1}[0,1]$.

$\left(\mathrm{H}_{2}\right) \mathrm{g}(\mathrm{x}, \mathrm{t})$ is continuous with respect to $x$ for all $t \in[0,1]$.

$\left(\mathrm{H}_{3}\right)$ There exist distributions $k, h \in \mathrm{D}_{\mathrm{HK}+}$, such that

$$
-k\|x\|-h \leqslant g(x, \cdot) \leqslant k\|x\|+h, \quad \forall x \in B_{r},
$$

where $B_{r}=\left\{x \in C^{1}[0,1] \mid\|x\|_{\infty} \leqslant r\right\}, r>0$.

$\left(\mathrm{H}_{4}\right) \mathrm{f} \in \mathrm{D}_{\mathrm{HK}}$.

Lemma 3.1. Under the assumptions $\left(\mathrm{H}_{1}\right)-\left(\mathrm{H}_{4}\right)$, the problem (1.1)-(1.2) is equivalent to the integral equation

$$
\begin{aligned}
x(t)= & \frac{t+\beta}{2} \int_{0}^{1} f(s)+g(s, x(s)) d s \\
& +\frac{t+\beta}{2} \int_{0}^{\eta} f(s)+g(s, x(s)) d s \\
& -\int_{0}^{t}(t-s)(f(s)+g(s, x(s))) d s, \quad t \in[0,1],
\end{aligned}
$$

where $\beta, \eta$ are constants with $0 \leqslant \eta \leqslant 1$.

Proof. For all $t \in[0,1], s \in[0,1], x \in \mathrm{C}^{1}[0,1], x^{\prime} \in \mathrm{C}[0,1]$, we have

$$
\int_{0}^{t} s x^{\prime \prime}(s) d s=\int_{0}^{t} s d x^{\prime}(s)=t x^{\prime}(t)-x(t)+x(0)
$$

Then

$$
x(t)=t x^{\prime}(t)+x(0)-\int_{0}^{t} s x^{\prime \prime}(s) d s
$$

Rewriting the differential equation (1.1) as $x^{\prime \prime}(t)=-f(t)-g(t, x(t))$, and integrating once over $[0, t]$, we obtain

$$
x^{\prime}(t)=x^{\prime}(0)-\int_{0}^{t}(f(s)+g(s, x(s))) d s .
$$

Combining with the boundary conditions (1.2), one has

$$
x^{\prime}(0)=\frac{1}{2} \int_{0}^{1} f(s)+g(s, x(s)) d s+\frac{1}{2} \int_{0}^{\eta} f(s)+g(s, x(s)) d s,
$$

and

$$
x(0)=\frac{\beta}{2} \int_{0}^{1} f(s)+g(s, x(s)) d s+\frac{\beta}{2} \int_{0}^{\eta} f(s)+g(s, x(s)) d s
$$


It follows from (3.2) and (3.3) that

$$
x(t)=t x^{\prime}(0)+x(0)-\int_{0}^{t}(t-s)(f(s)+g(s, x(s))) d s .
$$

Therefore, by (3.4), (3.5), (3.6), one has

$$
\begin{aligned}
x(t)= & \frac{t+\beta}{2} \int_{0}^{1} f(s)+g(s, x(s)) d s+\frac{t+\beta}{2} \int_{0}^{\eta} f(s)+g(s, x(s)) d s \\
& -\int_{0}^{t}(t-s)(f(s)+g(s, x(s))) d s, \quad t \in[0,1] .
\end{aligned}
$$

It is easy to calculate that the problem (1.1)-(1.2) holds by taking the derivative both sides of (3.1). This completes the proof.

Now we present the well-known Leray-Schauder nonlinear alternative theorem.

Lemma 3.2 ([7]). Let $\mathrm{F}$ be a Banach space and $\Omega$ a bounded open subset of $\mathrm{F}, 0 \in \Omega$. T : $\bar{\Omega} \rightarrow \mathrm{F}$ be a completely continuous operator. Then, either there exist $x \in \partial \Omega, \lambda>1$ such that $\mathrm{T}(\mathrm{x})=\lambda \mathrm{x}$, or there exists a fixed point $x^{*} \in \bar{\Omega}$.

With the help of the preceding two lemmas, we can now prove the existence of solutions of the problem (1.1)-(1.2).

Theorem 3.3. Suppose that the assumptions $\left(\mathrm{H}_{1}\right)-\left(\mathrm{H}_{4}\right)$ hold. If

$$
(|\beta|+4) \max _{t \in[0,1]}\left|\int_{0}^{t} k(s) d s\right|<1,
$$

then the problem (1.1)-(1.2) has at least one solution.

Proof. Let

$$
\begin{aligned}
& K(t)=\int_{0}^{t} k(s) d s, \\
& H(t)=\int_{0}^{t} h(s) d s, \\
& F(t)=\int_{0}^{t} f(s) d s, \quad t \in[0,1] .
\end{aligned}
$$

Then, by $\left(\mathrm{H}_{3}\right)$ and $\left(\mathrm{H}_{4}\right), \mathrm{K}, \mathrm{H}, \mathrm{F}$ are all continuous functions. Further,

$$
\max _{\mathrm{t} \in[0,1]}\left|\int_{0}^{\mathrm{t}} \mathrm{g}(\mathrm{s}, \mathrm{x}(\mathrm{s})) \mathrm{ds}\right| \leqslant\|\mathrm{K}\|_{\infty}\|\mathrm{x}\|_{\infty}+\|\mathrm{h}\|_{\infty} \leqslant \mathrm{r}\|\mathrm{K}\|_{\infty}+\|\mathrm{h}\|_{\infty} .
$$

Let

$$
\mathrm{r}=\frac{(|\beta|+4)\left(\|\mathrm{H}\|_{\infty}+\|\mathrm{F}\|_{\infty}\right)}{1-(|\beta|+4)\|\mathrm{K}\|_{\infty}}>0 .
$$

For each $x \in B_{r}, t \in[0,1]$, define the operator $\mathcal{T}: C[0,1] \rightarrow C[0,1]$ by

$$
\begin{aligned}
\mathcal{T} x(t):= & \frac{t+\beta}{2} \int_{0}^{1} f(s)+g(s, x(s)) d s \\
& +\frac{t+\beta}{2} \int_{0}^{\eta} f(s)+g(s, x(s)) d s \\
& -\int_{0}^{t}(t-s)(f(s)+g(s, x(s))) d s .
\end{aligned}
$$

Now we prove $\mathcal{T}$ is completely continuous in three steps. 
Step 1: $\mathcal{T}: \mathrm{B}_{\mathrm{r}} \rightarrow \mathrm{B}_{\mathrm{r}}$.

For all $x \in B_{r}$, by (3.8), one has

$$
\begin{aligned}
& \|\mathcal{T} x\|_{\infty}=\max _{\mathfrak{t} \in[0,1]} \mid \frac{\mathrm{t}+\beta}{2} \int_{0}^{1} f(s)+g(s, x(s)) d s \\
& +\frac{t+\beta}{2} \int_{0}^{\eta} f(s)+g(s, x(s)) d s \\
& -\int_{0}^{t}(t-s)(f(s)+g(s, x(s))) d s \mid \\
& \leqslant \max _{t \in[0,1]}(|\beta|+2)\left|\int_{0}^{t} f(s)+g(s, x(s)) d s\right| \\
& +\max _{t \in[0,1]}\left|\int_{0}^{t} s(f(s)+g(s, x(s))) d s\right| \\
& \leqslant(|\beta|+2)\left(r\|K\|_{\infty}+\|H\|_{\infty}+\|F\|_{\infty}\right) \\
& +\max _{t \in[0,1]}\left|\int_{0}^{t} s(f(s)+g(s, x(s))) d s\right| \text {. }
\end{aligned}
$$

Furthermore, let $G(t)=\int_{0}^{t} g(s, x(s)) d s, t \in[0,1]$. Then, by $\left(H_{1}\right), G$ is continuous on $[0,1]$. Therefore, for all $t \in[0,1]$, one has

$$
\begin{aligned}
\left|\int_{0}^{t} s(f(s)+g(s, x(s))) d s\right| & =\left|\int_{0}^{t} s d(F(s)+G(s))\right| \\
& =|s(F(s)+G(s))|_{0}^{t}-\int_{0}^{t} F(s)+G(s) d s \mid \\
& \leqslant|F(t)+G(t)|+\left|\int_{0}^{t} F(s)+G(s) d s\right| \\
& \leqslant 2\left(\|F\|_{\infty}+r\|K\|_{\infty}+\|H\|_{\infty}\right) .
\end{aligned}
$$

In view of (3.7), (3.9), (3.10), one has

$$
\|\mathcal{T} x\|_{\infty} \leqslant(|\beta|+4)\left(\mathrm{r}\|\mathrm{K}\|_{\infty}+\|\mathrm{H}\|_{\infty}+\|\mathrm{F}\|_{\infty}\right) \leqslant \mathrm{r} .
$$

Hence, $\mathcal{T}\left(\mathrm{B}_{\mathrm{r}}\right) \subseteq \mathrm{B}_{\mathrm{r}}$.

Step 2: $\mathcal{T}\left(B_{r}\right)$ is equicontinuous.

Let $t_{1}, t_{2} \in[0,1], x \in B_{r}$

$$
\begin{aligned}
\left|\mathcal{T} x\left(t_{1}\right)-\mathcal{T} x\left(t_{2}\right)\right|= & \mid \frac{t_{1}-t_{2}}{2} \int_{0}^{1} f(s)+g(s, x(s)) d s+\frac{t_{1}-t_{2}}{2} \int_{0}^{\eta} f(s)+g(s, x(s)) d s \\
& -\int_{0}^{t_{1}}\left(t_{1}-s\right)(f(s)+g(s, x(s))) d s \\
& +\int_{0}^{t_{2}}\left(t_{2}-s\right)(f(s)+g(s, x(s))) d s \mid \\
\leqslant & \left|t_{1}-t_{2}\right|\left(r\|K\|_{\infty}+\|H\|_{\infty}+\|F\|_{\infty}\right)+\left|\int_{t_{2}}^{t_{1}} F(s)+G(s) d s\right| \\
\leqslant & 2\left|t_{1}-t_{2}\right|\left(r\|K\|_{\infty}+\|H\|_{\infty}+\|F\|_{\infty}\right) \rightarrow 0, \text { as } t_{1} \rightarrow t_{2} .
\end{aligned}
$$

Then $\mathcal{T}\left(B_{r}\right)$ is equiuniformly continuous on $[0,1]$ for all $x \in B_{r}$.

In view of Steps 1 and 2 and Ascoli-Arzelà theorem, $\mathcal{T}\left(B_{r}\right)$ is relatively compact. 
Step 3: $\mathcal{T}$ is a continuous mapping.

Let $x \in B_{r},\left\{x_{n}\right\}_{n \in \mathbb{N}}$ be a sequence in $B_{r}$ and $x_{n} \rightarrow x$ as $n \rightarrow \infty$. By $\left(H_{2}\right)$, one has

$$
\mathrm{f}\left(\cdot, x_{n}\right) \rightarrow f(\cdot, x) \text { as } n \rightarrow \infty .
$$

According to the assumption $\left(\mathrm{H}_{3}\right)$ and Lemmas 2.5 and 2.8, we have

$$
\lim _{n \rightarrow \infty} \int_{0}^{t} g\left(s, x_{n}(s)\right) d s=\int_{0}^{t} g(s, x(s)) d s, \quad t \in[0,1],
$$

and

$$
\lim _{n \rightarrow \infty} \int_{0}^{t} s g\left(s, x_{n}(s)\right) d s=\int_{0}^{t} s g(s, x(s)) d s, \quad t \in[0,1] .
$$

On the other hand,

$$
\begin{aligned}
\mathcal{T} x_{n}(t)-\mathcal{T} x(t)= & \frac{\beta+\mathrm{t}}{2} \int_{0}^{1} g\left(s, x_{n}(s)\right)-g(s, x(s)) d s \\
& +\frac{\beta+t}{2} \int_{0}^{\eta} g\left(s, x_{n}(s)\right)-g(s, x(s)) d s \\
& +t \int_{0}^{t}\left(g\left(s, x_{n}(s)\right)-g(s, x(s))\right) d s \\
& +\int_{0}^{t} s\left(g\left(s, x_{n}(s)\right)-g(s, x(s))\right) d s, \quad t \in[0,1] .
\end{aligned}
$$

Therefore, $\lim _{n \rightarrow \infty} \mathcal{T} x_{n}()=.\mathcal{T} \chi($.$) , and hence \mathcal{T}$ is a completely continuous operator.

Finally, let $\Omega=\left\{x \in \mathrm{C}^{1}[0,1]:\|x\|<\mathrm{r}\right\}$. Assume that $x \in \partial \Omega, \lambda>1$ such that $\mathcal{T} x=\lambda x$. Then, by (3.11), one has

$$
\lambda r=\lambda\|x\|=\|\mathcal{T} x\| \leqslant r,
$$

which implies that $\lambda \leqslant 1$. This is a contradiction. Therefore, by Lemma 3.2, there exists a fixed point of $\mathcal{T}$ which is a solution of (3.1). By Lemma 3.1, the problem (1.1)-(1.2) has at least one solution.

If $k(t) \equiv 0$ on $[0,1]$, then $\left(\mathrm{H}_{3}\right)$ reduces into

$\left(\mathrm{H}_{3}^{\prime}\right)$ there exists a distribution $h \in \mathrm{D}_{\mathrm{HK}+}$, such that

$$
-h \leqslant g(x, \cdot) \leqslant h, \quad \forall x \in B_{r} .
$$

Therefore, we have the following result.

Corollary 3.4. Assume that $\left(\mathrm{H}_{1}\right),\left(\mathrm{H}_{2}\right),\left(\mathrm{H}_{3}^{\prime}\right),\left(\mathrm{H}_{4}\right)$ are fulfilled. Then the problem (1.1)-(1.2) has at least one solution.

Proof. This is a direct consequence of Theorem 3.3.

Remark 3.5. The condition $\left(\mathrm{H}_{3}^{\prime}\right)$ together with $\left(\mathrm{H}_{1}\right)$ and $\left(\mathrm{H}_{2}\right)$ was firstly proposed by Chew and Flordeliza [6] to deal with the first order Cauchy problems.

\section{Examples}

In this section, we shall give two examples for the applications of Theorem 3.3 and Corollary 3.4.

Example 4.1. Consider the initial value problem

$$
\left\{\begin{array}{l}
-x^{\prime \prime}=\frac{x \sin (x)}{3 \sqrt{5+t}}+\mathcal{R}^{\prime}, \quad t \in[0,1], \\
x(0)=2 x^{\prime}(0), \\
x^{\prime}(1)+x^{\prime}\left(\frac{1}{4}\right)=0,
\end{array}\right.
$$

where $\mathcal{R}^{\prime}$ is the distributional derivative of the Riemman function $\mathcal{R}(t)=\sum_{n=1}^{\infty} \frac{\sin n^{2} \pi t}{n^{2}}$ in [9]. 
Proof. It is easy to see that $\mathcal{R}(\mathrm{t}) \in \mathrm{C}[0,1]$ and $\mathcal{R}(0)=0$, hence $\mathcal{R}^{\prime}$ is $\mathrm{D}_{\mathrm{HK}}$-integrable. Let $\mathrm{f}=\mathcal{R}^{\prime}, \mathrm{g}(\mathrm{t}, \mathrm{x})=$ $\frac{x \sin x}{2 \sqrt{4+\mathrm{t}}}$, then $\left(\mathrm{H}_{1}\right),\left(\mathrm{H}_{2}\right),\left(\mathrm{H}_{4}\right)$ hold. Moreover, there exist HK integrable functions $k(t)=\frac{1}{3 \sqrt{5+\mathrm{t}}}, h(t)=1$ such that

$$
-k\|x\|-h \leqslant g(\cdot, x) \leqslant k\|x\|+h, \quad \forall x \in C[0,1],
$$

i.e., $\left(\mathrm{H}_{3}\right)$ holds. Let $\beta=2, \eta=\frac{1}{4}$, then

$$
(|\beta|+4) \max _{t \in[0,1]} \int_{0}^{t} \frac{1}{3 \sqrt{5+s}} \mathrm{ds}=4(\sqrt{6}-\sqrt{5}) \leqslant 1 .
$$

Since,

$$
0 \leqslant\|F\|_{\infty}=\|\mathcal{R}\|_{\infty} \leqslant \sum_{n=1}^{\infty} \frac{1}{n^{2}}=\frac{\pi^{2}}{6}
$$

by (3.7), we have,

$$
\begin{aligned}
40.98 \approx \frac{6}{1-4(\sqrt{6}-\sqrt{5})} & \leqslant r=\frac{(|\beta|+4)\left(\|\mathrm{H}\|_{\infty}+\|\mathrm{F}\|_{\infty}\right)}{1-(|\beta|+4)\|\mathrm{K}\|_{\infty}} \\
& \leqslant \frac{6+\pi^{2}}{1-4(\sqrt{6}-\sqrt{5})} \approx 108.46 .
\end{aligned}
$$

Therefore, by Theorem 3.3, problem (4.1) has at least one solution $x^{*}$ with

$$
\left\|x^{*}\right\| \leqslant \frac{6+\pi^{2}}{1-4(\sqrt{6}-\sqrt{5})} .
$$

Example 4.2. Consider the initial value problem

$$
\left\{\begin{array}{l}
-x^{\prime \prime}=\sin (x)+2 t \sin \left(t^{-2}\right)-\frac{2}{t} \cos \left(t^{-2}\right)+\mathcal{W}^{\prime}, \quad t \in[0,1] \\
x(0)=-\frac{1}{6} x^{\prime}(0) \\
x^{\prime}(1)+x^{\prime}\left(\frac{2}{3}\right)=0
\end{array}\right.
$$

where $\mathcal{W}^{\prime}$ is the distributional derivative of the Weierstrass function $\mathcal{W}(t)=\sum_{n=1}^{\infty} \frac{\sin 7^{n} \pi t}{2^{n}}$ in [9].

Proof. Obviously, $\mathcal{W}(t) \in C[0,1]$ and $\mathcal{W}(0)=0$, hence $\mathcal{W}^{\prime}$ is $D_{H K}$-integrable. Let $f=\mathcal{W}^{\prime}, g(t, x)=$ $\sin (x)+2 t \sin \left(t^{-2}\right)-\frac{2}{t} \cos \left(t^{-2}\right)$, then $\left(H_{1}\right),\left(H_{2}\right),\left(H_{4}\right)$ hold. Let

$$
k(t)=0, \quad h(t)=1+2 t \sin \left(t^{-2}\right)-\frac{2}{t} \cos \left(t^{-2}\right) .
$$

It is easy to see that the highly oscillating function $h(t)$ is HK integrable but not Lebesgue integrable, and

$$
H(t)=\int_{0}^{t} h(s) d s= \begin{cases}t+t^{2} \sin \left(t^{-2}\right), & t \in(0,1], \\ 0, & t=0 .\end{cases}
$$

Moreover, we have

$$
-h \leqslant g(\cdot, x) \leqslant h, \quad \forall x \in C[0,1],
$$

i.e., $\left(\mathrm{H}_{3}\right)$ holds. Let $\beta=-\frac{1}{6}, \eta=\frac{2}{3}$. Since,

$$
0 \leqslant\|F\|_{\infty}=\|\mathcal{W}\|_{\infty} \leqslant \sum_{n=1}^{\infty} \frac{1}{2^{n}}=1
$$




$$
\|\mathrm{H}\|_{\infty}=1+\sin (1)
$$

by (3.7), we have

$$
\begin{aligned}
7.67 \approx \frac{25}{6}(\sin (1)+1) & \leqslant r=\frac{(|\beta|+4)\left(\|\mathrm{H}\|_{\infty}+\|\mathrm{F}\|_{\infty}\right)}{1-(|\beta|+4)\|\mathrm{K}\|_{\infty}} \\
& \leqslant \frac{25}{6}(\sin (1)+2) \approx 11.84 .
\end{aligned}
$$

Therefore, by Corollary 3.4, problem (4.2) has at least one solution $x^{*}$ with

$$
\left\|x^{*}\right\| \leqslant \frac{25}{6}(\sin (1)+2) .
$$

Remark 4.3. It is worth pointing out that both the Riemman function $\mathcal{R}(t)$ and the Weierstrass function $\mathcal{W}(t)$ are continuous but pointwise differentiable nowhere on $[0,1]$, then the distributional derivatives $\mathcal{R}^{\prime}$ in (4.1) and $\mathcal{W}^{\prime}$ in (4.2) are neither HK integrable nor Lebesgue integrable. Hence, any result using HK or Lebesgue integral is not applicable for the case of Examples 4.1 and 4.2. In conclusion, the application of results in this paper is more extensive.

\section{Acknowledgment}

This research is supported by Educational Commission of Hubei Province (Grant No. B2016160), and the program of High-end Foreign Experts of the SAFEA (No. GDW 20163200216).

\section{References}

[1] B. Ahmad, A. Alsaedi, D. Garout, Monotone iteration scheme for impulsive three-point nonlinear boundary value problems with quadratic convergence, J. Korean Math. Soc., 45 (2008), 1275-1295. 1

[2] D. D. Ang, K. Schmitt, L. K. Vy, A multidimensional analogue of the Denjoy-Perron-Henstock-Kurzweil integral, Bull. Belg. Math. Soc. Simon Stevin, 4 (1997), 355-371. 2.2, 2.4, 2.5

[3] N. A. Asif, P. W. Eloe, R. A. Khan, Positive solutions for a system of singular second order nonlocal boundary value problems, J. Korean Math. Soc., 47 (2010), 985-1000. 1

[4] D.-L. Bai, H.-Y. Feng, Eigenvalue for a singular second order three-point boundary value problem, J. Appl. Math. Comput., 38 (2012), 443-452. 1

[5] P. S. Bullen, D. N. Sarkhel, On the solution of $(d y / d x)_{a p}=f(x, y)$, J. Math. Anal. Appl., 127 (1987), 365-376. 1

[6] T. S. Chew, F. Flordeliza, On $x^{\prime}=f(t, x)$ and Henstock-Kurzweil integrals, Differential Integral Equations, 4 (1991), 861-868. 3.5

[7] K. Deimling, Nonlinear functional analysis, Springer-Verlag, Berlin, (1985). 3.2

[8] P. W. Eloe, Y. Gao, The method of quasilinearization and a three-point boundary value problem, J. Korean Math. Soc., 39 (2002), 319-330. 1

[9] G. H. Hardy, Weierstrass's non-differentiable function, Trans. Amer. Math. Soc., 17 (1916), 301-325. 4.1, 4.2

[10] S.-M. Im, Y.-J. Kim, D.-I. Rim, A uniform convergence theorem for approximate Henstock-Stieltjes integral, Bull. Korean Math. Soc., 41 (2004), 257-267. 2

[11] R. A. Khan, Higher order nonlocal nonlinear boundary value problems for fractional differential equations, Bull. Korean Math. Soc., 51 (2014), 329-338. 1

[12] R. A. Khan, J. R. L. Webb, Existence of at least three solutions of a second-order three-point boundary value problem, Nonlinear Anal., 64 (2006), 1356-1366.

[13] M. K. Kwong, J. S. W. Wong, Solvability of second-order nonlinear three-point boundary value problems, Nonlinear Anal., 73 (2010), 2343-2352. 1

[14] P. Y. Lee, Lanzhou lectures on Henstock integration, Series in Real Analysis, World Scientific Publishing Co., Inc., Teaneck, NJ, (1989). 2

[15] S.-Q. Liang, L. Mu, Multiplicity of positive solutions for singular three-point boundary value problems at resonance, Nonlinear Anal., 71 (2009), 2497-2505. 1

[16] H.-S. Lü, C.-K. Zhong, Existence theorems for three-point boundary value problem, Ann. Differential Equations, 17 (2001), 162-172. 
[17] R.-Y. Ma, Existence theorems for a second order three-point boundary value problem, J. Math. Anal. Appl., 212 (1997), 430-442.

[18] R.-Y. Ma, Positive solutions for second-order three-point boundary value problems, Appl. Math. Lett., 14 (2001), 1-5. 1

[19] J. M. Park, J. J. Oh, C.-G. Park, D. H. Lee, The ap-Denjoy and ap-Henstock integrals, Czechoslovak Math. J., 57(132) (2007), 689-696. 2

[20] S. Schwabik, G.-J. Ye, Topics in Banach space integration, Series in Real Analysis, World Scientific Publishing Co. Pte. Ltd., Hackensack, NJ, (2005). 2

[21] Y.-P. Sun, L.-S. Liu, Solvability for a nonlinear second-order three-point boundary value problem, J. Math. Anal. Appl., 296 (2004), 265-275. 1

[22] Y.-P. Sun, M. Zhao, Existence of positive pseudo-symmetric solution for second-order three-point boundary value problems, J. Appl. Math. Comput., 47 (2015), 211-224. 1

[23] E. Talvila, The distributional Denjoy integral, Real Anal. Exchange, 33 (2008), 51-82. 2, 2.2, 2.3, 2, 2, 2.6, 2.7

[24] G.-J. Ye, W. Liu, The distributional Henstock-Kurzweil integral and applications, Monatsh. Math., 181 (2016), $975-989$. 2.1

[25] J.-F. Zhao, W.-G. Ge, A necessary and sufficient condition for the existence of positive solutions to a kind of singular three-point boundary value problem, Nonlinear Anal., 71 (2009), 3973-3980. 1 